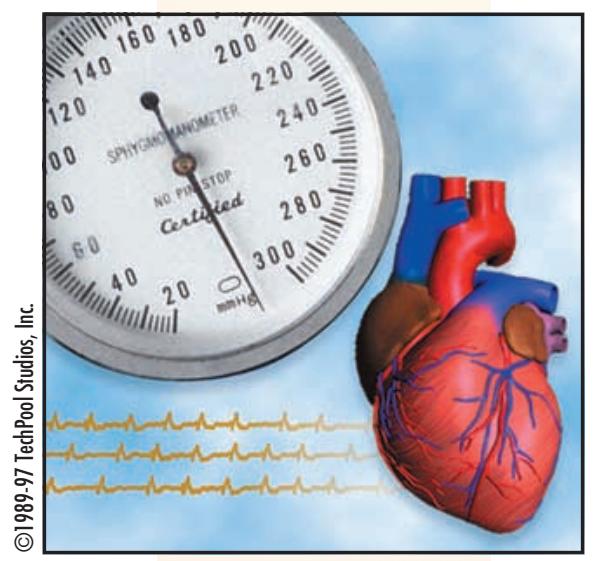

\title{
The Sympathetic Nervous System's Role in Regulating Blood Pressure Variability
}

\section{Understanding How SNA Controls Vascular Tone Is an Important Step in Determining the Pathogenesis of Hypertension}

\section{Simon C. Malpas, Bridget L. Leonard, Sarah-Jane Guild, John V. Ringwood, Michael Navakatikyan, Paul C. Austin, Geoffrey A. Head', Don E. Burgess ${ }^{2}$ Circulatory Control Laboratory, Departments of Physiology and Electrical and Electronic Engineering, University of Auckland \\ 'Baker Medical Research Institute, Melbourne \\ ${ }^{2}$ Department of Physiology, University of Kentucky}

ctivity of the sympathetic nervous system provides one of the fundamental mechanisms in the control of arterial pressure. By rapidly regulating the level of activity, sympathetic nerve activity (SNA) alters the degree of vasoconstriction in the blood vessels of many key organs in the body. This, in turn, increases or decreases blood flow through the organs, affecting both the function of these organs and arterial pressure. In contrast to the activity present in motor nerves, sympathetic nerves are continuously active, meaning that all innervated blood vessels remain under some degree of continuous constriction. Since its first description in the 1930s [1, 2], SNA has engendered itself to researchers in two camps; neurophysiologists have seen its inherent properties as an opportunity to understand how areas of the central nervous system may be connected to generate and control such activity [3-5], while cardiovascular physiologists have viewed its regulation of blood flow as a direct index of circulatory control in response to different stimuli, drugs, and pathological conditions [6-8].

There is now evidence from several animal models that sympathetic overactivity can initiate and/or subsequently maintain a blood pressure increase. In humans, essential hypertension is associated with elevated plasma noradrenaline levels, while muscle SNA is elevated in borderline hypertensives [9]. With regard to blood pressure variability, when one considers general variability using a simple standard deviation of blood pressure over 24 hours, the variability becomes progressively greater from normotensive to borderline, mild, and more severe essential hypertensive subjects [10]. While the mechanisms responsible for overall blood pressure variability are not yet defined, there is good evidence that the amplitudes of certain frequency bands strongly reflect the level of sympathetic drive. Understanding the origin and effect of these rhythms is likely to be of considerable clinical importance, as previous studies have shown altered blood pressure and heart rate variability to be associated with increased risk of cardiovascular mortality [11-13], raising the possibility of a diagnostic test using measurement of blood pressure and heart rate variability.

This article focuses on how SNA contributes to the variability seen in blood pressure. Specifically, it examines the following questions: why do oscillations occur at certain frequencies, why do only certain frequencies of oscillations in SNA induce oscillations in the vasculature, and what may be the functional purpose of these oscillations?

\section{The Cardiac- and Respiratory-Related Oscillations in SNA}

Postganglionic sympathetic nerves are composed of thousands of unmyelinated fibers [14], whose individual contributions to the recorded signal are exceedingly small. But fortunately, their ongoing activity can be measured from whole nerve recordings, because large numbers of fibers fire action potentials at almost the same time, to give discharges of summed spikes. For a detailed report of the nature and origin of the burst-like properties of SNA, readers are referred to a recent review [15].

Sympathetic activity can be thought of as a complex output of the central nervous 
system, providing subtle control over end organ function. This control is exerted in a number of frequency bands, including rhythms related to the cardiac and respiratory cycles, $10 \mathrm{~Hz}$, and between $0.2-0.4$ $\mathrm{Hz}$. The generation and control of each of these rhythms is likely to be quite separate. Although afferent feedback from sources such as baroreceptors can explain some of the rhythmical properties, in each case there is also good evidence for generation of aspects of these rhythms from central sources. The relevant aspect with regard to blood pressure is in understanding how these various rhythms set the level of blood pressure and regulate the amplitude of oscillations in blood pressure. With this understanding, it may be possible in the future to describe the changes in the control of blood pressure variability and thus identify early pathological changes before the onset of hypertension.

The most obvious rhythm present in direct recordings of postganglionic SNA is that related to the cardiac cycle (generally between $2-6 \mathrm{~Hz}$ in rats, rabbits, and cats). In spectral analysis of SNA, it is this frequency band that dominates, accounting for between $50-60 \%$ of the total power seen within the neurogram of rabbits [16], although to a lesser extent in rats [17]. While muscle SNA displays a much slower burst rate in humans, SNA still conforms to the same pattern of synchronized activation of many individual fibers at a certain phase of the cardiac cycle. Factors that govern the timing of these bursts are of some debate and beyond the scope of this review, other than to say the cardiac related discharges reflect inherent generation by a network of cell groups within the central nervous system and their entrainment by pulsatile input from arterial baroreceptor inputs. Readers are referred to a recent review specifically on this topic [18].

A respiratory-associated oscillation is the next most dominant rhythm present in SNA. Because blood pressure oscillates with a respiratory cycle, to some extent the strength and phase of the respiratory rhythm in SNA is a product of reflex modulation [19]. In other words, ventilation itself causes changes in intrathoracic pressure, which in turn affect venous return, cardiac output, and arterial pressure. This, in turn, activates both arterial and cardiopulmonary baroreceptors. However, such mechanically induced changes cannot account for differences among the rhythms of SNA to different end organs, some of which show little baroreceptor modulation; e.g., sudomotor [20]. Furthermore, it is known that artificial ventilation combined with a pneumothorax and vagotomy removes the afferent lung inflation signals and baroreceptor-mediated oscillation in arterial pressure, yet SNA still contains a respiratory signal that occurs out of phase with the ventilator [21]. The origin of inherent respiratory rhythm in SNA is thought to be due to a central coupling between respiratory neurons and neurons of autonomic pathways [22]. The magnitude of the respiratory modulation is proportional to respiratory drive. Hypercapnia enhances it, while hyperventilation decreases the sympathetic activation during inspiration [23, 24].

\section{How do the Cardiac- and Respiratory-Related Oscillations in SNA Influence the Vasculature?}

While blood pressure and blood flow display cardiac and respiratory related oscillations, this does not mean that these are induced by SNA. Instead they are rather simply due to the mechanical pumping nature of the heart and ventilation. Thus, an average sympathetic discharge rate between $2-6 \mathrm{~Hz}$ does not lead to a $2-6 \mathrm{~Hz}$ cycle of vasoconstriction and dilation in the vasculature, as the time constants for responses to changes in sympathetic activity at the neuromuscular junction are in the order of 1-25 s (see below) $[25,26]$. This raises the possible assumption that the respiratory- and cardiac-related rhythms in SNA simply reflect the processes of their generation, and by themselves do not influence the vasculature and therefore blood pressure. This is not correct: these rhythms in SNA are vitally important for contributing to a steady level of tone within the vasculature.

How then does one determine the specific role of the faster sympathetic rhythms in setting tone? One approach has been to measure how oscillations in blood pressure are transmitted through a particular organ; e.g., the kidney. The pressure signal may be dampened or amplified, and this is reflected in the calculated transfer function (gain) between blood pressure and renal blood flow (RBF). By comparing gain between animals with intact renal sympathetic nerves and animals who had undergone prior renal nerve denervation, it was shown that the gain was significantly reduced in the presence of SNA. This indicates that SNA acted to dampen the effect of changes in blood pressure on RBF [27]. In addition, the coherence between blood pressure and RBF was higher in renal denervated animals, indicating that in the absence of SNA there is a greater coupling between blood pressure and RBF. That is, the presence of SNA reduces the effect of rapid changes in arterial pressure on $\mathrm{RBF}$, thus altering the pressure-flow relationship.

One caveat in the above discussion is that while the cardiac and respiratory frequencies in SNA appear to contribute to vascular tone rather than inducing oscillations, one cannot assume that the synchronization of activity in individual axons has no purpose and that the same effect could be achieved by nonsynchronized unpatterned activity. Indirect evidence and theoretical studies suggest that such coordination leads to an increase in the gain of the system. That is, to have a signal where many thousands of nerve fibers are activated synchronously with a level of activation that may vary in both frequency and amplitude domains greatly increases the number of responses that can be configured to different stimuli. Birks [28] showed that electrical stimulation of preganglionic neurons with patterned stimulation, rather than constant frequency stimulation, increased the acetylcholine output of the terminals by as much as threefold. It was also shown that patterned stimuli assist the recruitment of a broader range of neurons than could be recruited by simple constant frequency stimuli [29]. The coordinated nature of the discharges may lead to a coordinated release of neurotransmitter at the neuromuscular junction $[30,31]$. In the rat caudal artery, burst patterning at a net frequency of $6 \mathrm{~Hz}$ resulted in a $44 \%$ greater contractile response than using equally spaced stimuli [32].

\section{An Integrating-Like Phenomena of the Vasculature}

Evidence that faster rhythms in SNA set tone within the vasculature and thus the level of blood pressure, rather than its absolute variability, is indicative of an integrating-like phenomena of the vasculature. (The lowpass filter effect dampens high frequencies and leaves the dc level, which sets the tone.) Although it is well established that the neuroeffector delays at vasculature smooth muscle have relatively long time constants, comparable to nerve conduction times or skeletomuscular 
neuroeffector junctions, until recently a precise frequency response profile of the vasculature response to SNA had not been defined. This response was clarified in a study of anesthetized rabbits in which the renal nerves were stimulated using modulated sine patterns (base frequency $5 \mathrm{~Hz}, 5$ ms duration pulses), which varied in amplitude between 0 and $10 \mathrm{~V}$ at a frequency between 0.04 and $1.0 \mathrm{~Hz}$. The strengths of the induced oscillations in RBF were calculated using spectral analysis. The faster rhythms in simulated SNA above $0.6 \mathrm{~Hz}$ contributed to the level of vascular tone, but only the slower frequency oscillations, less than $0.6 \mathrm{~Hz}$, induced an oscillation in RBF (Fig. 1). The overall frequency response curve is shown in Fig. 2, with 95\% of the power below $0.6 \mathrm{~Hz}$, indicating an integrating characteristic of the vasculature. The ability of an SNA rhythm at $0.6 \mathrm{~Hz}$ to induce a rhythm in RBF was 21 times less than that at $0.25 \mathrm{~Hz}$, if one calculates the oscillation amplitude as a percentage of the oscillation induced at $0.04 \mathrm{~Hz}$ (overall gain equaled $-20 \mathrm{~dB}$ per decade). It was also shown that sinusoidal stimulation at $0.16 \mathrm{~Hz}$ produced larger oscillations in RBF than at surrounding frequencies; i.e., the vasculature was more sensitive to SNA at this frequency, suggesting that SNA reveals resonance in the vasculature at this frequency. Such a phenomena of the renal vasculature has been observed using oscillations in blood pressure as the input [33-35], but it has not previously been considered that SNA could also evoke/reveal the same effects. Resonance is likely to result from a complex series of interactions among the characteristics of the neuro-muscular coupling, the characteristics of the second messenger pathways in the smooth muscle (i.e., the excitation-contraction coupling [25, 26, 36]), and the interaction with the intrinsic regulatory systems of the kidney (tubuloglomerular feedback and the myogenic response).

Other research groups have investigated the ability of the vasculature to respond to the different frequencies present in SNA. In an elegant study, Stauss et al. (37) electrically stimulated the paraventricular nucleus of the hypothalamus in conscious rats at frequencies ranging from 0.05 to $2.0 \mathrm{~Hz}$, while measuring splanchnic sympathetic nerve activity and mesenteric artery blood flow. This caused activation of SNA, with synchronous discharges up to stimulation frequencies of 2.0 Hz. Similar to the rabbit, the ability of the faster frequencies to induce an oscillation in mesenteric blood flow was negligible beyond stimulation frequencies of $1.0 \mathrm{~Hz}$.

It must also be acknowledged that all vascular beds may not necessarily have the same frequency-dependent response time. In a recent study in humans, using a unique form of acoustic stimuli to produce periodic sympathetic activation, Haynes et al. [38] observed that skin blood flow was most sensitive below $0.1 \mathrm{~Hz}$, suggesting that for this bed the frequency response curve is shifted to the left.

Another important piece of information to come from determining the frequency response characteristics of the vasculature is the phase relationship between SNA and the vasculature response (Fig. 2). While the phase difference shows a decrease with higher frequencies of stimulation, it also displays a portion that is frequency independent. This portion represents the pure time delay, and it reflects the total time (e.g., the release of neurotransmitter) from stimulation until the beginning of vasoconstriction. This time has been calculated at $1.13 \mathrm{~s}$ for the renal vasculature [39]. After subtracting this pure time delay, one is left with a dynamical time constant that varies with the frequency of stimulation, and it is more dominant at frequencies below $0.4 \mathrm{~Hz}$. Such information may be useful in model- ing the relationship between SNA and blood flow.

The characteristics of the filter response of the neuroeffector junction in the renal vasculature is such that higher SNA frequencies are effectively filtered and integrated to give most of the vasoconstrictor tone of the vasculature at a relatively steady level. Slower frequency oscillations in SNA $(<0.6 \mathrm{~Hz})$ induce oscillations in RBF at these same slower frequencies. The result of this duality appears to be to smooth the pulsatile impact of the blood pressure on RBF at the cardiac frequency, but to allow slower oscillations in SNA to be reflected in RBF. The question arises as to whether there is any inherent benefit to the kidney of such a biological filter. One might hypothesize that the maintenance of adequate glomerular filtration rate, and thus urine flow, requires $\mathrm{RBF}$ to remain relatively stable. If $\mathrm{RBF}$ responded to oscillations in SNA greater than $0.6 \mathrm{~Hz}$, this consistency of flow might be compromised. Conversely, a system that has no variability, and in which the inputs only adjust the mean level of RBF, may be have reduced controllability. Also the contribution of the renal bed in baroreflex control of the circulation would be limited, which would be undesirable for the short- to me-

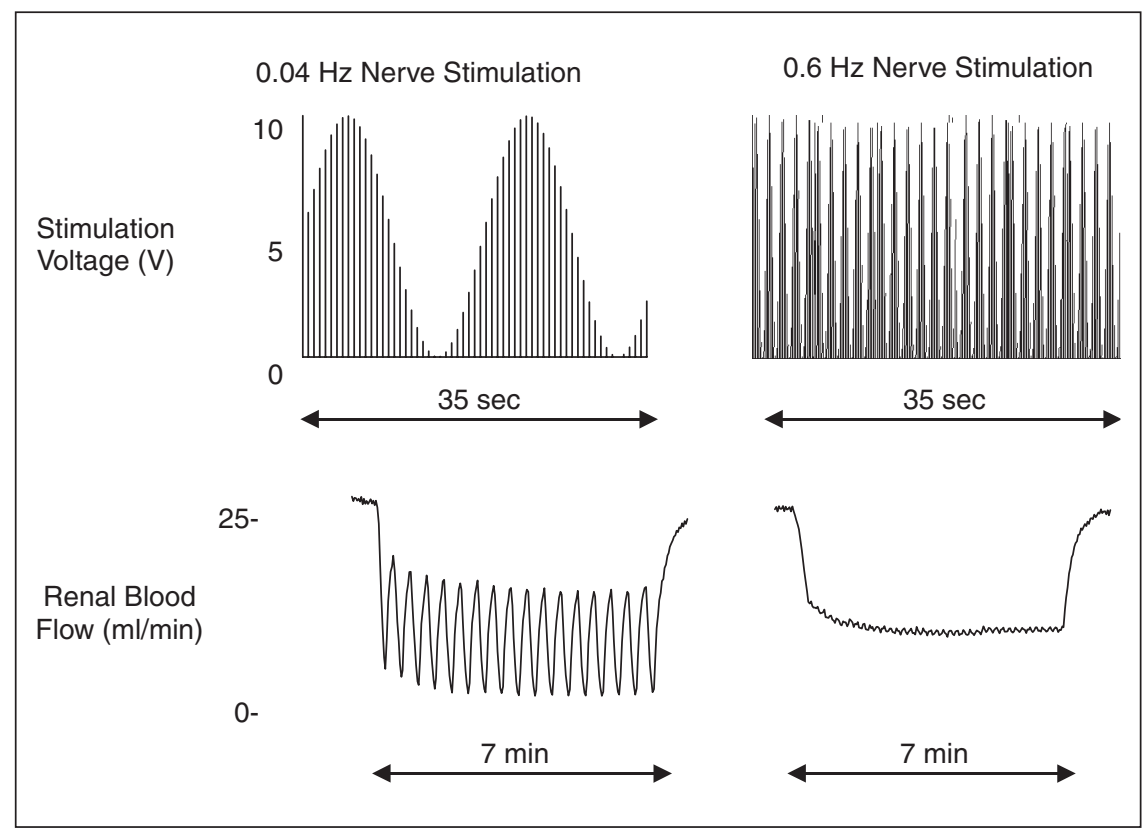

1. Examples of the effect of two sinusoidal stimulation sequences applied to the renal nerves on RBF ( $5 \mathrm{~Hz}$ base frequency using $5 \mathrm{~ms}$ pulses, see [39] for details). Note that some of the pulses at $0.04 \mathrm{~Hz}$ nerve stimulation have been removed for clarity. The amplitude of each of these pulses was varied in a sinusoidal fashion between 0 and $10 \mathrm{~V}$ at a predetermined frequency. It is clear that the faster frequency, while still producing a reduction in $\mathrm{RBF}$, did not produce an oscillation. Note that the stimulation sequence shown is for $35 \mathrm{~s}$, while the $\mathrm{RBF}$ response is from $7 \mathrm{~min}$. 


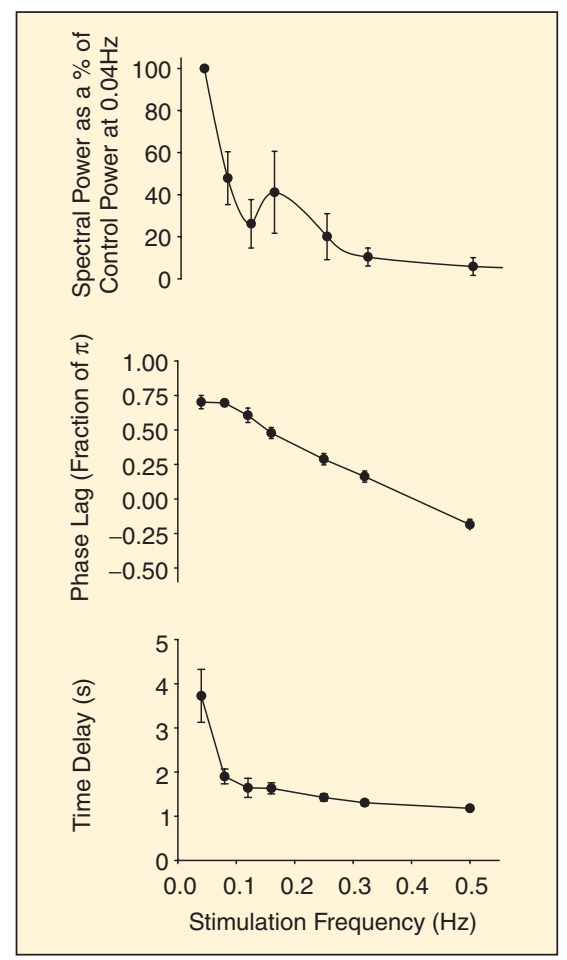

2. The mean frequency response curve (top panel: mean \pm SEM) for the $\mathrm{RBF}$ response to modulated sine patterns of electrical stimulation to the renal nerves. The amplitude of the induced oscillations was normalized to a percentage of the power of the oscillation at 0.04 Hz. Middle panel: the phase plot from the transfer function between nerve stimulation as the input and RBF as the output. This was converted to time delay (bottom panel) and illustrates a pure time delay that is independent of the frequency (mean $1.13 \mathrm{~s}$ ). dium-term (minutes) regulation of blood pressure. Therefore, the low-frequency oscillations in SNA may actually assist in the dynamic control of RBF, ensuring a rapid response with sufficiently high gain to the stimuli of daily life (Fig. 3). This dynamic control may also ensure a relatively stable flow within the renal microvasculature and therefore steady glomerular filtration rate, sodium excretion, and renin release.

\section{Oscillations in SNA Between 0.1 and $0.4 \mathrm{~Hz}$ and Their Link to Blood Pressure}

It is well established that blood pressure and SNA contain a distinct oscillation between 0.1 and $0.4 \mathrm{~Hz}$, depending on the species. In humans, this is $0.1 \mathrm{~Hz}$, and it is analogous to $0.3 \mathrm{~Hz}$ in rabbits and cats and $0.4 \mathrm{~Hz}$ in rats. The probable reason for the difference in this frequency between species is discussed below. It should be noted that this oscillation comprises only a small proportion of the total spectral power of all oscillations in SNA. With regard to renal SNA in conscious rabbits, the power in this band generally comprises no more than $15 \%$ of the overall power [16]. One could argue therefore that this rhythm is of little importance other than of esoteric interest. However, the main difference between the higher-frequency cardiac- and respiratory-related rhythms is that these low-frequency oscillations are slow enough to directly induce a rhythm of vasoconstriction and vasodilation in the smooth mus-

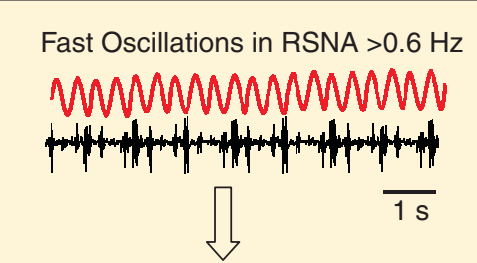

Tonic Constriction of Renal Arteries

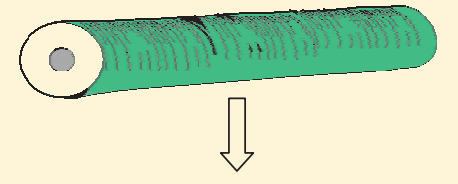

Dampens the Effect of Oscillations in Arterial Pressure on RBF

Enhances the Stability of RBF

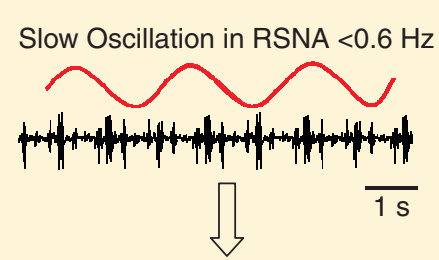

Cycles of Constriction and Dilation in Renal Vasculature

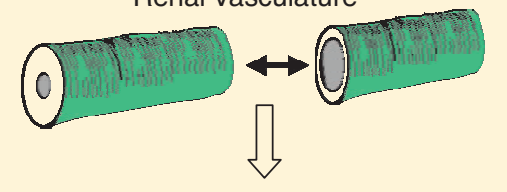

Slow Oscillations in RBF

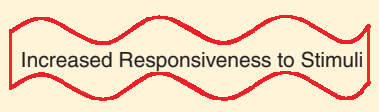

3. Schematic representation of the way different frequencies in SNA may control renal blood flow. Refer to text for explanation. cles of the vessels that the nerves innervate (see above). The end result of these oscillations is that blood pressure also contains a rhythm that is tightly linked to the same frequency [17]. Following from this is the suggestion that measurement of the strength of this oscillation in blood pressure may provide an index of SNA.

One of the potential pitfalls in measuring variability in blood pressure is in understanding that the absence of change does not necessarily mean an absence of an alteration in neural control. It is often overlooked that control of SNA is differential. Measurement of SNA to a single organ cannot be used to describe an effect of a treatment as increasing or decreasing global SNA [40, 41]. The SNA response to almost all stimuli is adjusted in a differential manner to different end organs, with quite clear differences among types of stimuli. In this way, a tailored response to each stimulus can be produced. For example, the response to moderate hypoxia in the rabbit is one of little change in blood pressure, which, however, belies a tremendous redistribution of blood flow [42]. SNA is profoundly increased to the kidney and gut, but decreased to the heart and skin [43]. It should be stressed that this differential control system is likely to provide a primary means of circulatory control in daily life. There is no reason to suggest that this differential control does not extend to the various oscillations present in SNA, where there may be increases in the strength of oscillations to the kidney, but decreases to other organs, with overall little effect on blood pressure variability (see below).

Since the development of a method for simultaneously recording of SNA and blood flow to the same kidney [16], it has been possible to analyze how the $0.3 \mathrm{~Hz}$ oscillation directly influences the vasculature. In rabbits, stimuli that increase the mean level of renal SNA, such as hypoxia and hemorrhage, have been shown to increase the strength of $0.3 \mathrm{~Hz}$ oscillations in both SNA and RBF $[16,27]$. Likewise, stimuli which decrease the mean level of SNA, such as plasma volume expansion, have been shown to decrease the strength of this oscillation (Fig. 4). Renal denervation abolishes this rhythm in RBF, providing it is not dominant in blood pressure. (A 0.2-0.4 Hz oscillation may be apparent in blood flow through sympathetic influences on other vascular beds, which will influence $\mathrm{RBF}$ 
in a direct pressure-flow relationship.) Taken together, these data indicate that oscillations in renal SNA are important in regulating oscillations in resting $\mathrm{RBF}$, i.e. oscillations in SNA help to regulate the variability of RBF.

In developing a procedure for quantifying SNA by measurement of blood pressure variability, it must be considered that not all the frequencies present below $0.5 \mathrm{~Hz}$ blood pressure may be driven by SNA. In a recent study, we observed that oscillations in blood flow and arterial pressure could occur at frequencies very close to the rhythm of neural origin, but quite distinct from it [27]. In conscious rabbits, hemorrhage was used to activate overall SNA, which led initially to an increase in oscillations at $0.3 \mathrm{~Hz}$ in renal blood flow. This rhythm could be directly ascribed to oscillations in SNA, as it did not occur in denervated rabbits (unless a $0.3 \mathrm{~Hz}$ oscillation also occurred in blood pressure via sympathetic effects on other end organs). However, as blood pressure began to fall, a new rhythm between $0.15-0.20 \mathrm{~Hz}$ became dominant in arterial pressure and renal blood flow in both intact and renal denervated rabbits. This rhythm also became apparent in SNA through arterial baroreflexes. This rhythm may reflect resonance within the vasculature that can be stimulated by the high circulating levels of angiotensin II or norepinephrine, which are known to occur in hemorrhage [44]. Importantly, the frequency of such a rhythm was very close to the $0.3 \mathrm{~Hz}$ oscillation normally induced by SNA. Thus, when measuring the spectral components of blood pressure under different conditions in humans, it is not possible to ensure that the increase in power at this frequency (approximately $0.1 \mathrm{~Hz}$ ) can be solely ascribed to an increase in SNA.

\section{The Origin of the Oscillation Between 0.1 and $0.4 \mathrm{~Hz}$}

The origin of this rhythm in SNA is still unresolved. Some authors have attributed it to an intrinsic central nervous system network [45, 46], although there seems little empirical evidence to support such a hypothesis. Current evidence and opinion seems to favor the concept of a resonant oscillation in the baroreflex loop [47-51]. In this model, a change in blood pressure is sensed by the arterial baroreceptors, altering the afferent signal to the central nervous system, and after some time constant ( $\tau_{1}$ in Fig. 5 ) this alters
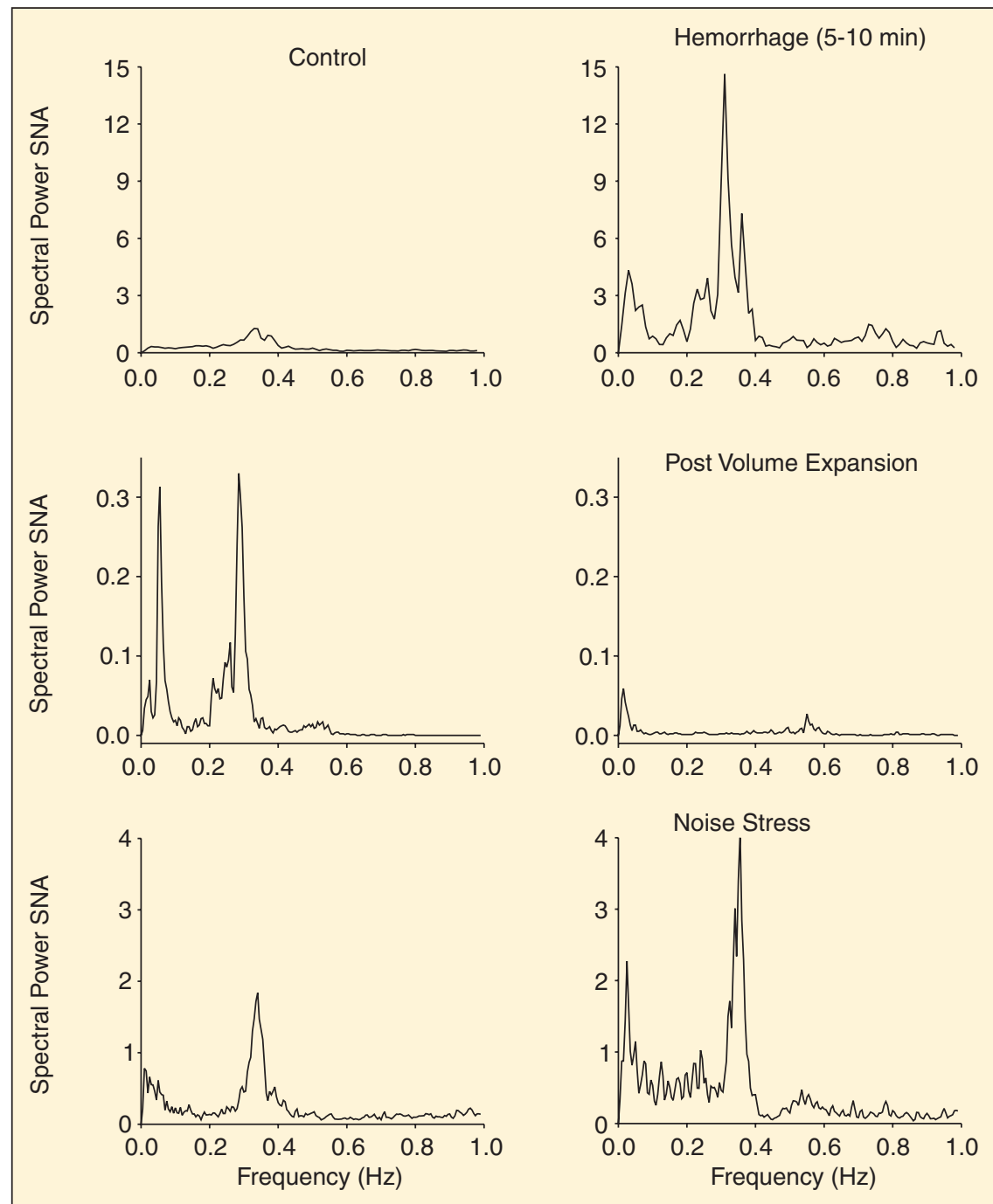

4. Power spectrums of SNA from individual conscious rabbits before and during hemorrhage $(1.35 \mathrm{ml} / \mathrm{min} / \mathrm{kg}$ for $10 \mathrm{~min}$, mean increase in SNA $21 \%$ [27]), after blood volume expansion $(1.5 \mathrm{ml} / \mathrm{min} / \mathrm{kg}$ for $15 \mathrm{~min}$, mean decrease in SNA $25 \%$ [62]), and during noise stress (mean increase in SNA 21\% [63]. Note the difference in the power of the $0.3 \mathrm{~Hz}$ SNA rhythm to rabbits under resting conditions. The 0.3 $\mathrm{Hz}$ rhythm in rabbits is analogous to the $0.1 \mathrm{~Hz}$ rhythm in humans and to $0.4 \mathrm{~Hz}$ in rats.

the SNA level. The resulting change in vascular tone in the target organ occurs after another delay, which is composed of a pure time-delay constant $\left(\tau_{2}\right.$ : this includes conduction velocity, neurotransmission, calcium release in the smooth muscle, etc.) and a dynamical time constant, T. This latter time constant will show up as frequency-dependent phase delay in a transfer function analysis (see above). Fundamental to this model is that the combination of these delays means that the input change in blood pressure results in an output change in vascular resistance that is slightly phase shifted and, instead of buffering the initial change in blood pressure, it leads to the develop- ment of its own change in blood pressure. Thus, the model is composed of an afferent arm and an efferent arm.

Fundamental to this model is the understanding that for an oscillation to occur in SNA, and thus in blood flow through that target organ, the SNA to that organ must be baroreceptor sensitive. It is well established that SNA to all organs does not display a uniform baroreceptor sensitivity. Lung, renal, and splenic SNA are highly baroreceptor sensitive [52, 53], displaying bursts of synchronized activity in a cardiac-related fashion (a hallmark of baroreceptor modulation [18]). However, SNA to the skin and gut are only weakly regulated by baroreceptor activity 


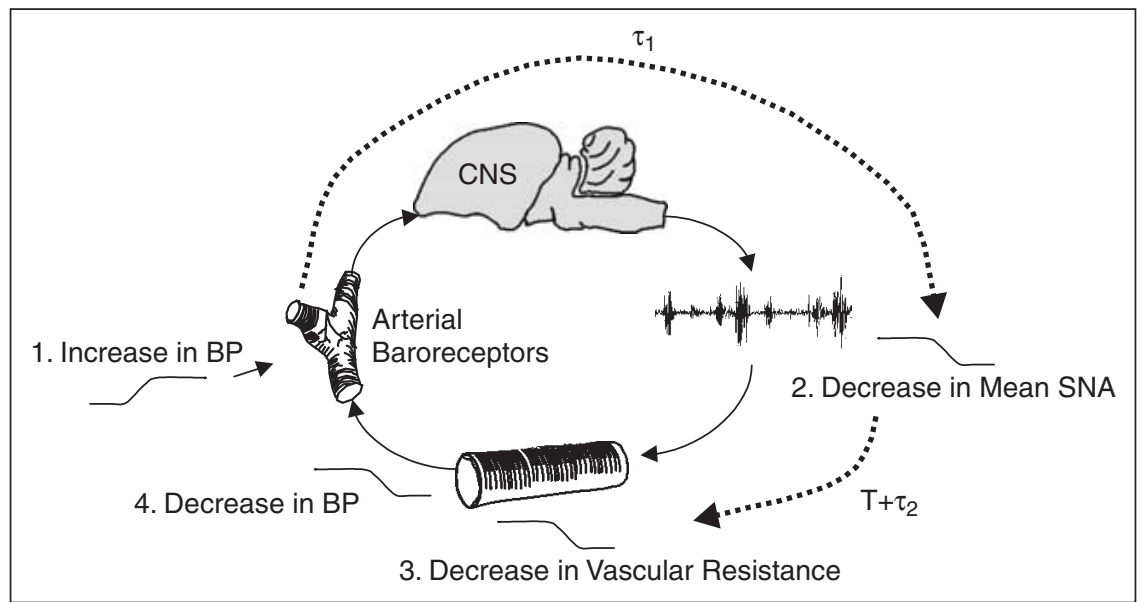

5. Schematic explanation accounting for the origin of the oscillation in SNA and blood pressure between 0.1 and $0.4 \mathrm{~Hz}$, depending on the species. $\tau_{1}$ refers to the time delay between baroreceptor sensing of the increase in blood pressure and the resulting decrease in SNA. $\tau_{2}$ refers to the pure fixed time taken for the vasculature to respond to the decrease in SNA (this includes conduction velocity, neurotransmission, calcium release, etc.). $T$ refers to a dynamical time constant that varies according to a range of frequency dependent factors.

[53-55]. Thus, we hypothesize that it is SNA to a few key organs, in particular the kidney, that dominates in the production of these oscillations. This does not mean that SNA to other organs does not play a role in regulating blood pressure but rather that their role is more confined to the steady state (dc gain) control of blood pressure, rather than variability in the 0.1-0.4 Hz range. In support of this hypothesis, if all organs contributed to the origin of the oscillations in this frequency range in proportion to their percentage of cardiac output, then it is suggested that no oscillation would be evident, as the slow conduction velocities in postganglionic nerves $(0.7 \mathrm{~m} / \mathrm{s}$ [56]) would mean that SNA to the extremities would reach its target up to $1 \mathrm{~s}$ later than the innervation to major organs in the thoracic cavity. This delay would result in the vasoconstriction response to the SNA signal being nonuniform, which should not be misinterpreted to indicate that blood flow to nonkey organs (e.g., skin) would not display a $0.1-0.4 \mathrm{~Hz}$ oscillation (species dependent). An oscillation at this frequency in blood pressure would drive an oscillation in blood flow.

Another aspect of the oscillations between $0.1-0.4 \mathrm{~Hz}$ that requires discussion is the difference in the frequency among species. While it is true that the frequency appears to be related to the resting heart rate, there is no evidence to suggest that this is anything other than an association. Rather, it is more likely that the larger dis- tance between arterial baroreceptors, the brain, and the target organ in man as compared to rabbits and rats, means that conduction times - a component of $\tau_{1}$ and $\tau_{2}$ in Fig. 5-accounts for the difference.

\section{Future Directions}

While overactivity of the sympathetic nervous system has been indicated in a number of pathologies, including heart failure [57], cirrhosis of the liver [58], and in the initiation and development of some types of hypertension [59-61], it remains unknown whether the various rhythms in SNA may also be altered. The question is also whether these changes are the cause or are the result of the increase in blood pressure. One possibility is that chronic changes in specific SNA frequencies may occur in some pathologies.

There is a need to establish long-term recordings of SNA in conscious animals to test the hypothesis that a chronic change in the SNA oscillatory processes can be the initiator or a contributor to a pathological series of events; that is, that altered SNA, perhaps without necessarily an increase in its mean level, can act as an initiator for altered control of end organ function and ultimately lead to the development of hypertension. Given that recordings can be made in conscious rabbits for up to three weeks, this species seems the ideal candidate. Such experiments would go some way to defining the role of the various frequencies present in SNA in the long term regulation of arterial pressure.

\section{Acknowledgments}

Work in the authors' laboratory is supported by the Auckland Medical Research Foundation, the Marsden Fund, the Health Research Council, and the Lottery Grants Board of New Zealand.

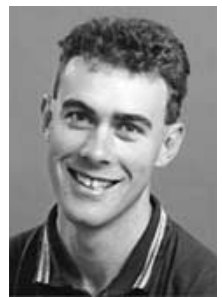

Simon Malpas received his B.Sc. (Hons) in physiology from Victoria University, Wellington, New Zealand in 1986 and a Ph.D. from the University of Otago, Dunedin, New Zealand, in 1990. This was followed by postdoctoral research at the $\mathrm{Na}-$ tional Cardiovascular Research Center, Osaka, Japan; the Department of Physiology, University of Birmingham, United Kingdom; and at the Baker Medical Research Institute, Melbourne, Australia. He currently holds the position of senior lecturer and head of the Circulatory Control Laboratory in the Department of Physiology, University of Auckland, New Zealand. Simon's research interests focus on the how the sympathetic nervous system controls blood pressure. A particular focus is on the dynamic relationship between sympathetic activity, renal blood flow, and blood pressure.

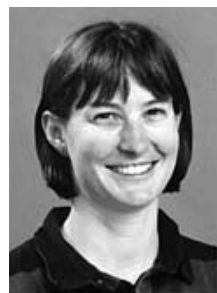

Bridget Leonard graduated from the University of Otago, New Zealand, with a B.Sc. (Hons) in physiology from University of Otago, New Zealand, in 1994. She is currently working on her Ph.D. in the Departments of Physiology at the University of Auckland, New Zealand. This work is being completed under the supervision of Dr. Simon Malpas.

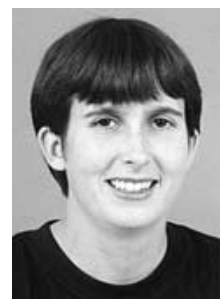

Sarah-Jane Guild graduated from the University of Auckland, New Zealand, with an M.E. (Eng. Sci.) in 1999 having obtained her B.E. (Eng. Sci.) two years before. She is currently working on her Ph.D. in the departments of Electrical and Electronic Engineering and Physiology at the University of Auckland, New Zealand. This work is being done under the supervision of associate professor Paul Austin and Dr. Simon Malpas. 


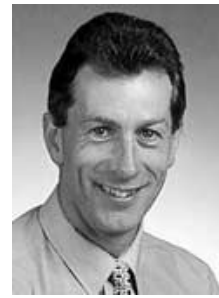

John V. Ringwood graduated from Dublin Institute of Technology, Ireland, with the B.Sc. (Eng) in 1981 and a $\mathrm{Ph} . \mathrm{D}$. from the University of Strathclyde, Scotland, in 1985. Prior to his appointment as professor and head of the Dept. of Electronic Engineering at NUI Maynooth, John was a senior lecturer at Dublin City University and has held visiting appointments at Massey University and the University of Auckland in New Zealand. His research interests focus on the development of advanced control strategies over a range of industrial applications, including the dairy industry and mathematical modeling and forecasting, particularly for electrical utilities. He has also a keen research interest in modeling of physiological systems and analysis of feedback effects in such systems. John is a chartered engineer and a Fellow of the Institution of Electrical Engineers.

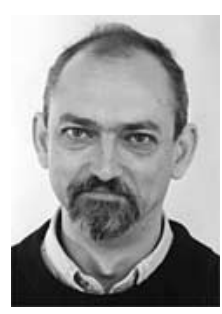

Michael Navakatikyan received his M.Sc. in human and animal physiology from Kiev State University, Kiev, Ukraine, in 1974 and his Ph.D. from the Institute of Higher Nervous Activity and Neurophysiology of the Academy of Science of the USSR, now the Russian Academy of Science, Moscow, in 1981. This was followed by the work in the Ukrainian Scientific Hygienic Centre of Health Ministry, Ukraine, as senior researcher, and since 1991 as chief of the Applied Physiology and Biochemistry Research Laboratory. He currently holds a postdoctoral position in the Circulatory Control Laboratory of the Department of Physiology, University of Auckland, New Zealand. His scientific interests lie in the areas of circulatory control, behavioral effect of the electromagnetic fields, mathematical modeling of the psychological processes, and neural control of the kidney circulation.

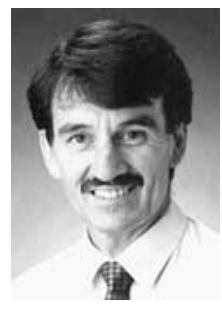

Paul Austin is an associate professor in the Department of Electrical and Electronic Engineering, University of Auckland, New Zealand.

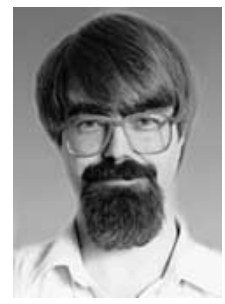

Geoffrey A. Head received his B.Sc.(Hons) in pharmacology from the University of Melbourne, Australia, in 1976 and a Ph.D. from Monash University, Australia, in 1981. Following a postdoctorate position at the Rudolf Magnus Institute of Pharmacology, Utrecht, The Netherlands, and the National Institute of Health, Bethesda, Maryland, USA, he returned to the Baker Medical Research Institute, Melbourne, Australia, in 1985. He currently holds the position of principle research fellow as the head of the Neuropharmacology Laboratory at the Baker Institute, and he is an honorary associate professor with the Department of Pharmacology at Monash University. Geoff's research interests include the understanding of mechanisms involved in the control of the heart and circulation by the central nervous system, including the action of centrally acting antihypertensive agents, baroreflex mechanisms in hypertension, CNS control of renal sympathetic nerve activity, spectral analysis, and radiotelemetry.

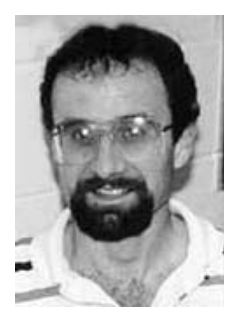

Don E. Burgess graduated from Pennsylvania State University, USA, in 1980. Following graduation, he served in the United States Navy for three years. In 1990, he graduated from Ohio State University with a Ph.D. in physics. Subsequently, he did his postdoctoral research at the Center for Nonlinear Studies at the Los Alamos National Laboratory. He is currently an associate professor of physics at Asbury College in Wilmore, Kentucky, and a staff research assistant at the University of Kentucky. His research interests include oscillations in physiological control systems, the nature of "slow" fluctuations in blood pressure, and kinetic modeling of ion channels.

Address for Correspondence: Dr. Simon C. Malpas, Circulatory Control Laboratory, Department of Physiology, University of Auckland Medical School, Private Bag 92019, Auckland, New Zealand. Fax: 64-9-3737499. E-mail; s.malpas@auckland.ac.nz.

\section{References}

1. Adrian ED, Bronk DW, and Phillips G: Discharges in mammalian sympathetic nerves. $J$ Physiol 74: 115-133,1932

2. Bronk DW, Ferguson LK, Margaria R, and Solant DY: The activity of the cardiac sympathetic centers. Am J Physiol 117: 237-249,1936

3. Kocsis B, Fedina L, Gyimesi-Pelczer K, Ladocsi T, and Pasztor E: Differential sympathetic reactions during cerebral ischemia in cats: The role of desynchronized nerve discharge. $J$ Physiol 469: 37-50,1993

4. McAllen RM and May CN: Brainstem neurones and postganglionic sympathetic nerves: Does correlation mean connection? Acta Neurobiol Exp 56(1): 129-135, 1996.

5. Gebber GL: Basis for phase relations between baroreceptor and sympathetic nervous discharge. Am J Physiol 230(2): 263-70, 1976.

6. Dibona GF, Jones SY, and Sawin LL: Renal sympathetic neural mechanisms as intermediate phenotype in spontaneously hypertensive rats. Hypertension 27(3 Part 2): 626-630, 1996.

7. Mark AL: Regulation of sympathetic nerve activity in mild human hypertension. $J$ Hypertens 8(Suppl.7): S67-S75, 1990.

8. Head GA, Burke SL, and Chan CKS: Central imidazoline receptors and centrally acting anti-hypertensive agents. Clin Exp Hypertens 19(5-6): 591-605, 1997.

9. Goldstein DS: Plasma norepinephrine during stress in essential hypertension. Hypertension 3(5): 551-6, 1981.

10. Mancia G, Ferrari A, Gregorini L, Parati G, Pomidossi G, Bertinieri G, Grassi G, di Rienzo M, Pedotti A, and Zanchetti A: Blood pressure and heart rate variabilities in normotensive and hypertensive human beings. Circ Res 53(1): 96-104,1983.

11. Farrell TG, Bashir Y, Cripps T, Malik M, Plooniecki J, Bennett ED, Ward DE, and Camm AJ: Risk stratification for arrhythmic events in postinfarction patients based on heart rate variability, ambulatory electrocardiographic variables and the signal-averaged electrocardiogram. J Am Coll Cardiol 18: 687-697, 1991.

12. Mancia G, Giannattasio C, Turrini D, Grassi G, and Omboni S: Structural cardiovascular alterations and blood pressure variability in human hypertension. J Hypertension 13(Suppl. 2): S7-S14, 1995.

13. Parati G, Ravogli A, Frattola A, Groppelli A, Ulian L, Santucciu C, and Mancia G: Blood pressure variability: Clinical implications and effects of antihypertensive treatment. $J$ Hypertension 12: 535-540, 1994.

14. DiBona GF, Sawin LL, and Jones SY: Differentiated sympathetic neural control of the kidney. Am J Physiol 271(1): R84-R90, 1996.

15. Malpas SC: The rhythmicity of sympathetic nerve activity. Prog Neurobiol 56(1): 65-96, 1998.

16. Janssen BJA, Malpas SC, Burke SL, and Head GA: Frequency-dependent modulation of renal blood flow by renal nerve activity in conscious rabbits. Am J Physiol 273(2): R597-608, 1997. 
17. Brown DR, Brown LV, Patwardhan A, and Randall DC: Sympathetic activity and blood pressure are tightly coupled at $0.4 \mathrm{~Hz}$ in conscious rats. Am J Physiol 267: R1378-R1384, 1994.

18. McAllen RM and Malpas SC: Sympathetic burst activity: Characteristics and significance. Clin Exp Pharmacol Physiol 24: 791-799, 1997.

19. Boczek-Funcke A, Dembowsky K, Habler HJ, Jänig W, McAllen RM, and Michaelis M: Classification of preganglionic neurones projecting into the cat cervical sympathetic trunk. $J$ Physiol 453(319): 319-39, 1992.

20. Jänig W: Pre- and postganglionic vasoconstrictor neurons: Differentiation, types, and discharge properties. Ann Rev Physiol 50:525-539, 1988.

21. Miyawaki T, Pilowsky P, Sun QJ, Minson J, Suzuki S, Arnolda L, Llewellynsmith I, and Chalmers J: Central inspiration increases barosensitivity of neurons in rat rostral ventrolateral medulla. Am $J$ Physiol 268:R909-R918, 1995.

22. Pilowsky P: Good vibrations? Respiratory rhythms in the central control of blood pressure. Clin Exp Pharmacol Physiol 22(9): 594-604, 1995.

23. Cohen MI and Gootman PM: Periodicities in efferent discharge of splanchnic nerve of the cat. Am J Physiol 218: 1092-1101, 1970.

24. Boczek-Funcke A, Dembowsky K, Habler HJ, Jänig W, and Michaelis M: Respiratory-related activity patterns in preganglionic neurones projecting into the cat cervical sympathetic trunk. J Physiol 457(277): 277-96, 1992.

25. Hirst GD and Edwards FR: Sympathetic neuroeffector transmission in arteries and arterioles. Physiol Rev 69(2): 546-604, 1989.

26. Somlyo AP and Somlyo AV: Flash photolysis studies of excitation-contraction coupling, regulation, and contraction in smooth muscle. Ann Rev Physiol 52: 857-874, 1990.

27. Malpas SC, Evans RG, Head GA, and Lukoshkova EV: Contribution of renal nerves to renal blood flow variability during hemorrhage. Am J Physiol 274: R1283-R1294, 1998.

28. Birks RI: Regulation by patterned preganglionic neural activity of transmitter stores in a sympathetic ganglion. $J$ Physiol 280: 559-572, 1978.

29. Birks RI, Laskey W, and Polosa C: The effect of burst patterning of preganglionic input on the efficacy of transmission at the cat stellate ganglion. J Physiol 318: 531-539, 1981.

30. Stjarne L and Stjarne E: Geometry, kinetics and plasticity of release and clearance of ATP and noradrenaline as sympathetic cotransmitters: Roles for the neurogenic contraction. Prog Neurobiol 47(1): 45-94,1995.

31. Bao JX: Sympathetic neuromuscular transmission in rat tail artery: A study based on electrochemical, electrophysiological and mechanical recording. Acta Physiol Scand Suppl 610: 1-58, 1993.

32. Hardebo JE: Influence of impulse pattern on noradrenaline release from sympathetic nerves in cerebral and some peripheral vessels. Acta Physiol Scand 144(3): 333-339, 1992.
33. Cupples WA, Novak P, Novak V, and Salevsky FC: Spontaneous blood pressure fluctuations and renal blood flow dynamics. Am $J$ Physiol 270(1 Pt 2): F82-9, 1996.

34. Just A, Wittmann U, Ehmke H, and Kirchheim H: Autoregulation of renal blood flow in the conscious dog and the contribution of the tubuloglomerular feedback. J Physiol 506(1): 275-290, 1998.

35. Holstein Rathlou NH, and Marsh DJ: A dynamic model of renal blood flow autoregulation. Bull Math Biology 56(3): 411-29, 1994.

36. Hirst GDS, Bramich NJ, Edwards FR, and Klemm M: Transmission at autonomic neuroeffector junctions. Trends in Neuroscience 15(2): 40-46, 1992.

37. Stauss HM, Persson PB, Johnson AK, and Kregel KC: Frequency-response characteristics of autonomic nervous system function in conscious rats. Am J Physiol 273: H786-H795, 1997.

38. Haynes WG, Stauss HM, Kregel KC, Khendoguina E, and Anderson EA: Modulation of sympathetic effector organs by acoustic stimulations in humans. FASEB J 13: A450, 1999. 39. Malpas SC, Hore TA, Navakatykyan M, Lukoshkova EV, Nguang SK, and Austin P: Resonance in the renal vasculature evoked by activation of the sympathetic nerves. Am J Physiol 276: R1311-R1319, 1999.

40. Morgan DA, Anderson EA, and Mark AL: Renal sympathetic nerve activity is increased in obese zucker rats. Hypertension 25(4 Part 2): 834-838, 1995.

41. Somers VK, Dyken ME, Clary MP, and Abboud FM: Sympathetic neural mechanisms in obstructive sleep apnea. J Clin Invest 96(4): 1897-1904, 1995.

42. Korner P and Uther J: Dynamic characteristic of the cardiovascular autonomic effects during severe arterial hypoxia in the unanesthetized rabbit. Circ Res 24(May): 671-687, 1969.

43. Iriki M, Kozawa E, Korner P, and Dorward P: Arterial and cardiopulmonary baroreceptor and chemoreceptor influences and interaction on ear sympathetic nerve discharge in the rabbit. Jap J Physiol 29: 551-558, 1979.

44. Korner PI, Oliver JR, Zhu JL, Gipps J, and Hanneman F: Autonomic, hormonal, and local circulatory effects of hemorrhage in conscious rabbits. Am J Physiol 258: H229-H239, 1990.

45. Koepchen HP, Abel HH, and Kusseldorf D: Central cardiorespiratory organization. In: Lown B, Malliani A, Prosdocimi M(Eds). Neural Mechanisms and Cardiovascular Disease. Vol. 5. Padua: Liviana Press, pp. 119-131, 1986.

46. Haxhiu MA, Van Lunteren E, Deal EC, and Cherniack NS: Role of the ventral surface of the medulla in the generation of Mayer waves. Am J Physiol 257: R804-R809, 1989.

47. DeBoer R, Karemaker J, and Strackee J: Hemodynamic fluctuations and baroreflex sensitivity in humans: a beat-to-beat model. Am J Physiol 253:680-689, 1987.

48. Burgess DE, Hundley JC, Li SG, Randall DC, Brown DR: First-order differential-delay equation for the baroreflex predicts the $0.4-\mathrm{Hz}$ blood pressure rhythm in rats. Am J Physiol 42(6): R1878-R1884, 1997.
49. Wessling KH and Settels JJ: Baromodulation explains short term blood-pressure variability. In: Orlebeke JF, Mulder J, VanDoornen LJP, (Eds). Psychophysiology of Cardiovascular Control. New York: Plenum, pp. 69-97, 1985.

50. Madwed J, Albrecht P, Mark R, and Cohen R: Low-frequency oscillations in arterial pressure and heart rate: A simple computer model. Am J Physiol 256: H1573-H1579, 1989.

51. Bernardi L, Leuzzi S, Radaelli A, Passino C, Johnston JA, and Sleight P: Low-frequency spontaneous fluctuations of R-R interval and blood pressure in conscious humans: A baroreceptor or central phenomenon? Clin Sci 87(6): 649-654, 1994.

52. Shirai M, Matsukawa K, Nishiura N, and Ninomiya I: Effects of baroreceptor reflex on efferent pulmonary sympathetic nerve activity in anesthetized cat. Am J Physiol 37(4): R1078-R1083, 1995.

53. Ninomiya I, Nisimaru $\mathbf{N}$, and Irisawa $\mathbf{H}$ : Sympathetic nerve activity to spleen, kidney, and heart in response to baroreceptor input. Am J Physiol 221: 1346-1351, 1971.

54. Nishimaru N: Comparison of gastric and renal nerve activity. Am J Physiol 220: 1303-1308, 1971.

55. Ninomiya I and Fujita S: Reflex effects of thermal stimulation on sympathetic nerve activity to skin and kidney. Am J Physiol 230(2): 271-278, 1976.

56. Dorward PK, Burke SL, Jänig W, and Cassell J: Reflex responses to baroreceptor, chemoreceptor and nociceptor inputs in single renal sympathetic neurones in the rabbit and the effects of anaesthesia on them. J Auton Nerv Syst 18: 39-54, 1987.

57. Dibona GF and Sawin LL: Increased renal nerve activity in cardiac failure: arterial vs cardiac baroreflex impairment. Am J Physiol 268: R112-R116, 1995.

58. Dibona GF and Sawin LL: Hepatorenal baroreflex in cirrhotic rats. Am J Physiol 269(1): G29-G33, 1995.

59. Floras JS and Hara K: Sympathoneural and haemodynamic characteristics of young subjects with mild essential hypertension. J Hypertension 11: 647-655, 1993.

60. Lucini D, Pagani M, Mela GS, and Malliani A: Sympathetic restraint of baroreflex control of heart period in normotensive and hypertensive subjects. Clin Sci 86: 547-556, 1994.

61. Anderson EA, Sinkey CA, Lawton WJ, and Mark AL: Elevated sympathetic nerve activity in borderline hypertensive humans: Evidence from direct intraneural recordings. Hypertension 14: 177-183, 1989.

62. Leonard BL, Navakatikyan MA, Wilton ER, and Malpas SC: Effect of volume expansion on rhythm in renal nerve activity: does renal blood flow follow the beat? FASEB J 13: A456, 1999.

63. Malpas SC and Evans RG: Do different levels and patterns of sympathetic activation all provoke renal vasoconstriction? J Auton Nerv Syst 69(1): 72-82, 1998. 